\title{
Phase diagram of silicon from atomistic simulations
}

\author{
M. Kaczmarski, ${ }^{1,2}$ O. N. Bedoya-Martínez, ${ }^{1}$ and E. R. Hernández ${ }^{1, *}$ \\ ${ }^{1}$ Institut de Ciència de Materials de Barcelona (ICMAB-CSIC), Campus de Bellaterra, 08193 Barcelona, Spain \\ ${ }^{2}$ Institute of Physics, University of Silesia, Uniwersytecka 4, 40-007 Katowice, Poland
}

(Dated: July 7, 2018)

\begin{abstract}
In this letter we present a calculation of the temperature-pressure phase diagram of $\mathrm{Si}$ in a range of pressures covering from -5 to $20 \mathrm{GPa}$ and temperatures up to the melting point. The phase boundaries and triple points between the diamond, liquid, $\beta$-Sn and $\mathrm{Si}_{34}$ clathrate phases are reported. We have employed efficient simulation techniques to calculate free energies and to numerically integrate the Clausius-Clapeyron equation, combined with a tight binding model capable of an accuracy comparable to that of first-principles methods. The resulting phase diagram agrees well with the available experimental data.
\end{abstract}

PACS numbers: 64.30.+t, 64.70.-p, 65.40.-b

Temperature-pressure phase diagrams charter the regions of stability of the different allotropes of a material. The confection of phase diagrams has been a long standing objective of experimental physics, chemistry and materials science. However, to date, the phase diagrams of most materials remain relatively unknown beyond the domain of normal conditions, because of the technical challenge of performing accurate phase behavior studies in conditions of extreme temperatures and/or pressures. Reliable first principles electronic structure calculations [1] have the potential to be of great assistance in this problem, and indeed they have proved their value with impressive demonstrations of their capabilities, such as the calculation of the melting curve of iron down to the pressure regime of the Earth's core [2], that of aluminum [3], or or that of hydrogen in a similar range of pressures [4]. But such calculations, which employ either free energy evaluation techniques like thermodynamic integration, or directly address phase coexistence by explicitly simulating the interface, are computationally demanding, and by no means routine. The twophase method, in particular, requires large simulation cells where the two phases can be monitored in coexistence, and it is only directly applicable to solid-liquid equilibria. Nevertheless, in recent years several simulation techniques have been developed which now make free energy calculations [5, 6] and phase boundary determination 7] much more accessible. In this letter we demonstrate the potential of these novel techniques by using them to obtain, entirely from atomistic simulations, the phase diagram of $\mathrm{Si}$ in a wide range of temperatures and pressures.

In spite of being one of the most extensively studied materials, the phase diagram of $\mathrm{Si}$ is not accurately known. As many as eleven phases other than diamond have been identified at high pressures [8], with at least six of them being thermodynamically stable in some temperature-pressure domain. It is now also clearly established [9] that other phases become stable at negative pressures: the so called clathrate phases [10], of which
$\mathrm{Si}_{34}$ (also known as $\mathrm{Si}_{136}$ ) has interesting semiconducting properties [11] of its own. In $\mathrm{Si}$, as in many other materials, computer simulations have been a great aid in identifying, and sometimes even predicting [12, 13. the occurrence of certain phases, but they have not been much used to help establishing the limits of stability of the different phases except at zero temperature 14] or zero pressure 15, 16]. In this letter we calculate the phase diagram of Si entirely from atomistic simulations, for temperatures ranging between 0 and $1700 \mathrm{~K}$, and for pressures in the range -5 to $20 \mathrm{GPa}$. We have considered four different phases in this temperature-pressure region, namely the diamond structure (Si I), the $\beta$-Sn phase (II), $\mathrm{Si}_{34}(\mathbf{C})$ and the liquid phase $(\mathbf{L})$, and provide the five corresponding coexistence curves between these, as well as estimates for the location of the two triple points to be found in this area of the phase diagram.

In a recent study [17, we have shown that certain tight binding [18] models, such as those of Kwon et al. 19] and Lenosky et al. 20], are capable of providing very accurate descriptions not only of the structural properties of $\mathrm{Si}$, but more importantly of the thermal properties too. In fact, these two models predict a melting temperature at zero pressure which is in better agreement with the experimental value than that provided by first principles calculations 15, 16]. The deciding factor between the Kwon and Lenosky models is that only the latter correctly predicts a pressure-induced transition from the $\mathbf{I}$ to II phase [20]. All calculations reported below were carried out using supercells containing $128 \mathrm{Si}$ atoms, except in the case of Si $\mathbf{C}$, where a supercell of 136 atoms was imposed by the structure. Four special k-points [21] were used to sample the Brillouin zone and provided a sufficient degree of convergence even for the metallic phases (II and $\mathbf{L}$ ). All calculations were performed with the Trocadero code 22.

Let us now briefly describe the procedure adopted for determining the phase diagram. Firstly, for all pairs of phases for which a phase boundary is sought, a coexistence point along the boundary must be found, i.e. a tem- 


\begin{tabular}{|c|c|c|c|}
\hline Phase boundary & $\begin{array}{c}\text { Pressure } \\
(\mathrm{GPa})\end{array}$ & $\begin{array}{c}\text { Temperature } \\
(\mathrm{K})\end{array}$ & $\begin{array}{c}\mathrm{dT} / \mathrm{dP} \\
\left(\mathrm{K} \mathrm{GPa}^{-1}\right)\end{array}$ \\
\hline $\mathbf{I}-\mathbf{L}$ & 0 & $\begin{array}{c}1551 \pm 66 \\
\left(1687^{a}\right)\end{array}$ & $\begin{array}{c}-16 \\
\left(-33^{a}\right)\end{array}$ \\
\hline II-L & 10 & $1230 \pm 25$ & 40 \\
\hline C-L & 0 & $\begin{array}{c}1424 \pm 57 \\
\left(1473^{b}\right) \\
\end{array}$ & -73 \\
\hline I-II & $\begin{array}{c}15.5 \pm 0.4 \\
\left(10.4-12.4^{c}\right)\end{array}$ & $\begin{array}{c}500 \\
\left(573^{c}\right)\end{array}$ & -200 \\
\hline I-C & $-2.47 \pm 0.03$ & 500 & $\approx 10^{4}$ \\
\hline
\end{tabular}

TABLE I: Coexistence points obtained between the different phases considered in this study. Experimental data is given in parenthesis when available. These points were later used as starting initial conditions for dynamical Clausius-Clapeyron integration. The local slope of the corresponding coexistence line is also given.

perature and pressure for which the Gibbs free energies of the two phases in question are equal. If the coexistence line has a small (in absolute value) pressure derivative, in order to locate the coexistence point it is better to fix the pressure of both phases at some convenient value, $P$, and to calculate the Gibbs free energy of each phase in a temperature interval bracketing the coexistence temperature, $T_{c}$, at fixed pressure. This can be done employing the reversible scaling technique of de Koning et al. [6]. If, on the other hand, the phase boundary is expected to have a large (in absolute value) pressure derivative, as is common in solid-solid coexistence lines, it is more convenient to fix the temperature and to monitor the Gibbs free energy of each phase as a function of pressure, which can be done with the adiabatic switching technique [5]. Once a coexistence point has been thus located, the rest of the phase boundary is obtained by solving numerically the Clausius-Clapeyron equation:

$$
\frac{d T_{c}}{d P}=T_{c} \frac{\Delta V}{\Delta H}
$$

where $T_{c}$ is the coexistence temperature at pressure $P$, and $\Delta V$ and $\Delta H$ are the difference of volumes and enthalpies of the two phases, respectively. This was done using the dynamical scheme of de Koning and coworkers 7]. All the above techniques require to simulate the system under isothermal-isobaric conditions, and this was done as described by Hernández [23]. Following the scheme outlined above, we proceeded to locate initial coexistence points along the phase boundaries between the diamond-liquid (I-L), $\beta$-Sn-liquid (II-L), clathrate-liquid $(\mathbf{C}-\mathbf{L})$, diamond-clathrate $(\mathbf{I}-\mathbf{C})$ and diamond- $\beta$-Sn $(\mathbf{I}-\mathbf{I I})$ phases. Further calculations were carried out at the found coexistence points in order to quantify the errors incurred in our estimations of those conditions. For coexistence temperatures at fixed pressure the error can be estimated from $\delta T_{c} \approx \delta G / \Delta S[24]$, where $\delta G$ is the error in the Gibbs free energy difference, and $\Delta S$ is the entropy change. For coexistence pressures at fixed temperature, the error can be estimated from $\delta P \approx \delta G / \Delta V$, where $\Delta V$ is the change of volume. As well as error estimates, these calculations allowed us to obtain the values of the pressure derivatives of each phase boundary at the coexistence point from Eq. (1). Table (II) lists the locations of the different coexistence points, together with their estimated errors and the local pressure derivatives of the corresponding coexistence lines.

Our calculated zero-pressure melting point for Si-I $(1551 \mathrm{~K})$ is in reasonably good agreement with the accepted experimental value of $1687 \mathrm{~K}[8]$. Although the difference between these values may appear to be large, we note that density functional theory (DFT) calculations using the local density approximation (LDA) for the exchange-correlation energy predict values in the range 1300-1350 K 15, 16], and $1492 \pm 50 \mathrm{~K}$ when a generalized-gradient approximation (GGA) is used instead [16]. Comparison with our own previous estimation using the same model [17] gives a difference of $30 \mathrm{~K}$, within our error bars for the melting temperature, although we regard the present value as more accurate. In agreement with experiments and with DFT calculations we obtain a negative slope for the phase boundary. The melting of (metastable) Si-II at $10 \mathrm{GPa}$ is found to occur at $1230 \mathrm{~K}$. This temperature is already higher than the experimental estimate of the $\mathbf{I}-\mathbf{I I}-\mathbf{L}$ triple point temperature 25], but is consistent with our own prediction for this triple point (see below). Like the $\mathbf{I}$ phase, $\mathbf{C}$ has a melting line with a negative slope. For this phase we predict a melting temperature at zero pressure of $1424 \mathrm{~K}$, which is only $50 \mathrm{~K}$ below the experimentally measured value at this pressure [9], and also in good agreement with the calculated value of Wilson \& McMillan [27]. As for the coexistence pressure between Si-I and Si-II at $500 \mathrm{~K}, 15.5 \mathrm{GPa}$, it is larger than the experimental values, 10.4-12.4 GPa at $573 \mathrm{~K} 25$, but we note again that DFT-LDA calculations predict a value of $8 \mathrm{GPa}$ 14] at zero temperature, while recent quantum Monte Carlo calculations place it at $16.5 \mathrm{GPa}$ 28]. At the same temperature, the $\mathbf{I}-\mathbf{C}$ coexistence point is found at $-2.47 \mathrm{GPa}$, in good agreement with experimental measurements [9] and with both empirical potential simulations 27] and first principles calculations [26].

Taking as starting conditions the coexistence points thus located, we then proceeded to run dynamical Clausius-Clapeyron [7] integration calculations, thereby obtaining the sought phase boundaries. Fig. (1) shows our calculated phase diagram for $\mathrm{Si}$, and constitutes the central result of this work. For comparison, Fig. (11) also shows a summary of recent experimental observations from Voronin et al. 25], Hu et al. [30] and McMillan [9]. As can be seen, the calculated phase diagram captures 
all the main features of the experimental data with surprising fidelity. Nevertheless, there are differences in the details which are mostly attributable to minor shortcomings in the Lenosky tight-binding model.

The $\mathbf{I}-\mathbf{L}$ coexistence line has, as previously mentioned, a negative pressure derivative, which increases slightly toward larger negative values as the pressure is raised. This is in agreement with experimental observations by Voronin et al. 25] that suggest this behavior, although the pressure derivative predicted by the Lenosky model for this phase boundary is too small. An independent error estimate of the melting line away from the starting point of the dynamical Clausius-Clapeyron integration calculation was obtained at a point of coordinates $T=1385 \mathrm{~K}$ and $P=8 \mathrm{GPa}$. We found an uncertainty of $95 \mathrm{~K}$, which is not significantly worse than that of the zero-pressure melting point. The $\mathbf{I}-\mathbf{L}$ melting line meets the $\mathbf{I}-\mathbf{I I}$ phase boundary at a temperature $T \approx 1290 \mathrm{~K}$ and $P \approx 10.9 \mathrm{GPa}$, according to our results. The experimental coordinates of this triple point are not accurately known, though a recent estimate by Voronin et al. [25] puts it at $T=1003 \pm 20 \mathrm{~K}$ and $P=10.5 \pm 0.2 \mathrm{GPa}$. Compared with this best experimental estimate, our triple point temperature is somewhat too high (by nearly $300 \mathrm{~K}$ ), consistent with the fact that $d T_{c} / d P$ for the $\mathbf{I}-\mathbf{L}$ melting line is too small in absolute value. However, we emphasize that the estimate of Voronin et al. is a lower bound; if one assumes that the $\mathbf{I}-$ $\mathbf{L}$ value of $d T / d P$ remains constant and equal to its zero pressure value, then at $10.5 \mathrm{GPa}$ the triple point temperature should be $1340 \mathrm{~K}$, which is much closer to our figure. Thus, it is very likely that our triple point temperature and that of Voronin et al. provide upper and lower bounds respectively for the true value. The agreement in the value of the pressure coordinate is much better (in fact, within our error estimate for the I-II phase boundary), but must be understood as somewhat fortuitous, resulting from error cancellation between a I-II coexistence pressure at $500 \mathrm{~K}$ which is slightly too high, and a value of $d T_{c} / d P$ for the $\mathbf{I}-\mathbf{I I}$ phase boundary which is most likely smaller than the experimental one. Nevertheless, it should be pointed out that, to our knowledge, this is the first prediction from atomistic simulations of the location of the of the $\mathbf{I}-\mathbf{I I}-\mathbf{L}$ triple point of $\mathrm{Si}$. We have also calculated the $\mathbf{I I}-\mathbf{L}$ phase boundary, starting from a temperature and pressure where both the II and $\mathbf{L}$ phases are metastable. Indeed, the II-L coexistence line crosses the $\mathbf{I}-\mathbf{I I}$ and $\mathbf{I}-\mathbf{L}$ boundaries very close to the point where the $\mathbf{I}-\mathbf{L}$ and $\mathbf{I}-\mathbf{I I}$ boundaries cross, a good indication of the internal consistency of our calculations.

The I-II phase boundary has, as expected, a large pressure derivative, of negative sign. Experimental observations 25] seem to agree with this finding, although data is only available at large temperature intervals, and it is not possible at present to compare with an experimental value of the pressure derivative. In any case,

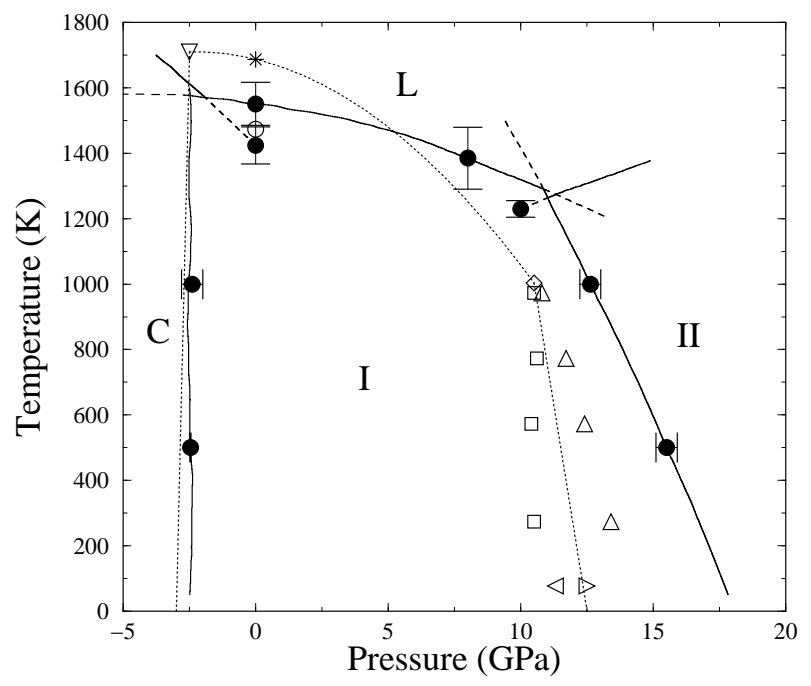

FIG. 1: Silicon phase diagram. The continuous and dashed lines indicate the calculated phase diagram. Dashed curves indicate phase boundaries in regions where the separated phases are metastable, while continuous black curves separate thermodynamically stable phases; uncertainty bounds estimated at specific points of the phase diagram (marked by filled circles) are provided by the error bars. For comparison purposes, a schematic phase diagram summarizing the experimental data is shown with dotted lines, and experimental data at specific temperatures and pressures is shown in the form of empty symbols. The asterisk corresponds to the zeropressure melting point of phase $\mathbf{I}, 1687 \mathrm{~K}$ [8]; the circle is the zero-pressure melting point of the (metastable) $\mathbf{C}$ phase, at $1473 \mathrm{~K} \mathrm{[9]}$; the diamond is the I-II-L triple point, with estimated coordinates of $1003 \pm 20 \mathrm{~K}$ and $10.5 \pm 0.2 \mathrm{GPa}$ 25]; empty squares and triangles indicate the pressures at which the II phase was first observed and where the I phase ceased to be detected, respectively, in the experiments of Voronin et al. [25]; left and right pointing triangles give the same information as obtained by $\mathrm{Hu}$ et al. [30]; finally, the downward pointing triangle is the estimated $\mathbf{I}-\mathbf{C}-\mathbf{L}$ triple point, at $1710 \mathrm{~K}$ and $-2.5 \mathrm{GPa}$ [9].

it can be concluded that, as it happened with the $\mathbf{I}-\mathbf{L}$ melting curve, the pressure derivative of the I-II coexistence line is probably not as large as the experimental one. This can be seen from the fact that both coexistence pressures at $T=500 \mathrm{~K}$ and in the limit $T \rightarrow 0 \mathrm{~K}$ are several GPa higher than the experimental coexistence pressures at similar temperatures, while our pressure coordinate for the $\mathbf{I}-\mathbf{I I}-\mathbf{L}$ triple point agrees quite well with the experimental value. It is worth noting that the coexistence pressure at $T \rightarrow 0 \mathrm{~K}$ deduced from the calculated I-II phase boundary $(18.1 \mathrm{GPa})$ is very close to the value obtained by calculating the enthalpies of both phases at $T=0 \mathrm{~K}(18.5 \mathrm{GPa})$, which serves as another internal consistency check for our calculations. To further validate our results, we performed an error estimation of the coexistence pressure at $1000 \mathrm{~K}$. Our results indicate that 
the coexistence pressure predicted by the phase boundary at that temperature $(12.6 \mathrm{GPa})$ is accurate to within $0.4 \mathrm{GPa}$.

The $\mathbf{I}-\mathbf{C}$ phase boundary we have obtained is nearly a vertical line at $P \approx-2.5 \mathrm{GPa}$. At $500 \mathrm{~K}$ our calculated coexistence point occurred at a pressure of $-2.47 \pm$ $0.02 \mathrm{GPa}$ [see Table (I)]. At $1000 \mathrm{~K}$, we calculated a second coexistence point to double check the phase boundary calculation, obtaining a pressure of $-2.4 \pm 0.4 \mathrm{GPa}$, to be compared with a value of $-2.5 \mathrm{GPa}$ according to our calculated phase boundary. The pressure derivative of this phase boundary is so large $\left(\approx 10^{4} \mathrm{~K} / \mathrm{GPa}\right.$ in absolute value) as to make it virtually impossible to predict its sign with any accuracy. This is a manifestation of the fact that at coexistence, not only the Gibbs free energies of the two phases are the same, but also their enthalpies are nearly equal. This in turn implies that the entropies of the $\mathbf{I}$ and $\mathbf{C}$ phases are very similar at coexistence conditions. The $\mathbf{I}-\mathbf{C}$ and $\mathbf{I}-\mathbf{L}$ boundaries cross at $T \approx 1576 \mathrm{~K}$ and $P \approx-2.48 \mathrm{GPa}$, marking within our error bars the coordinates of the $\mathbf{I}-\mathbf{C}-\mathbf{L}$ triple point. Wilson \& McMillan 27] have estimated the location of this triple point to be $1750 \mathrm{~K}$ and $-1.5 \mathrm{GPa}$, from two-phase coexistence calculations employing the Stillinger-Weber potential [29]. Experimental estimates 9] suggest that the triple point may actually be closer to $1710 \mathrm{~K}$ and $-2.5 \mathrm{GPa}$. Again, we note that our predicted temperature coordinate is too low by about $100-150 \mathrm{~K}$, consistent with our underestimation of the $\mathbf{I}$ melting temperature at zero pressure, while the pressure coordinate is closer to the experimental estimation. The $\mathbf{C}-\mathbf{L}$ melting line is almost straight, with a negative slope of $-73 \mathrm{~K} / \mathrm{GPa}$; it crosses the $\mathbf{I}-\mathbf{L}$ melting line slightly to the right of the point where the latter is crossed by the $\mathbf{I}-\mathbf{C}$ boundary, but their separation is within our estimated error bars.

Thus it is seen that, in spite of its simplicity and semiempirical nature, the Lenosky model provides a fairly good description of the phase diagram of Si, being probably as accurate as could be expected of first principles calculations. We have obtained five phase boundaries and two triple points between four phases of the silicon phase diagram, in reasonable agreement with the known experimental data. The simulation techniques employed in this study to calculate free energies and to obtain coexistence curves are straight forward and efficient, and can equally well be used in combination with first principles methods. It can be concluded, then, that the combination of techniques used here brings about the possibility of obtaining entire phase diagrams of complex materials completely $a b$ initio.

The use of computer facilities at $C^{4}$ (Centre de Computaciò i Comunicaciò de Catalunya), CESGA (CEntro de Supercomputación de GAlicia), ICM (Interdisciplinary Center for Mathematical and Computer Modeling, Warsaw University), and TASK (Academic Computer Center, Gdansk) is gratefully acknowledged. This work has been funded by the Spanish Ministry of Science and Education (MEC) under project BFM2003-03372C03.

* To whom correspondence should be addressed; email: ehe@icmab.es

[1] R. M. Martin, Electronic structure: basic theory and practical methods (Cambridge University Press, Cambridge 2004).

[2] D. Alfè, M. J. Gillan and G. D. Price, Nature 401462 (1999).

[3] L. Vočadlo and D. Alfè, Phys. Rev. B 65, 214105 (2002).

[4] S. A. Bonev, E. Schwegler, T. Ogitsu and G. Galli, Nature 431, 669 (2004).

[5] M. Watanabe and W. P. Reinhardt, Phys. Rev. Lett. 65, 3301 (1990).

[6] M. de Koning, A. Antonelli and S. Yip, Phys. Rev. Lett. 83, 3973 (1999).

[7] M. de Koning, A. Antonelli and S. Yip, J. Chem. Phys. 115, 11025 (2001).

[8] Hull, R. (Ed.) Properties of Crystalline Silicon (Inspec, London 1999); M. I. McMahon and R. J. Nelmes, Phys. Rev. B 47, 8337 (1993); M. I. McMahon, et al., Phys. Rev. B 50, 793 (1994); J. Z. Hu and I. L. Spain, Solid State Comm. 51, 263 (1984); H. Olijnyk et al., Phys. Lett. A 703, 137 (1984); J. Crain et al., Phys. Rev. B 50, 13043 (1994); R. O. Piltz et al. Phys. Rev. B 52, 4072 (1995).

[9] P. F. McMillan, Nature Materials, 1, 19 (2002).

[10] Clathrates were first synthesized in the 1960s, see C. Cros et al., C. R. Acad. Sci. Paris, 260, 4764 (1965); J. S. Kasper et al., Science 150, 11713 (1965).

[11] X. Blase, Phys. Rev. B 67, 035211 (2003).

[12] R. J. Needs and R. M. Martin, Phys. Rev. B 305390 (1984).

[13] K. J. Chang and M. L. Cohen, Phys. Rev. B 317819 (1985).

[14] R. J. Needs and A. Mujica, Phys. Rev. B 51, 9652 (1995).

[15] O. Sugino and R. Car, Phys. Rev. Lett. 74, 1823 (1995).

[16] D. Alfè and M. J. Gillan, Phys. Rev. B 68, 205212 (2003).

[17] M. Kaczmarski, R. Rurali, R. and E. R. Hernández, Phys. Rev. B 69, 214105 (2004).

[18] C. M. Goringe, D. R. Bowler and E. R. Hernández, Rep. Prog. Phys. 60, 1447 (1997).

[19] I. Kwon, R. Biswas, C. Z. Wang, K. M. Ho and C. M. Soukoulis, Phys. Rev. B 49, 7242 (1994).

[20] T. J. Lenosky, et al., Phys. Rev. B 55, 1528 (1997).

[21] H. J. Monkhorst and J. D. Pack, Phys. Rev. B 13, 5188 (1976).

[22] R. Rurali and E. R. Hernández, Comput. Mat. Sci. 28 85 (2003).

[23] E. R. Hernández, J. Chem. Phys. 115, 10282 (2001).

[24] G. A. de Wijs, G. Kresse and M. J. Gillan, Phys. Rev. B 57, 8223 (1998).

[25] G. A. Voronin, C. Pantea, T. W. Zerda, L. Wang and Y. Zhao, Phys. Rev. B. 68, 020102 (2003).

[26] Ramachandran, G. K. et al., J. Phys.: Condens. Matter 12, 4013 (2000); A. San Miguel et al., Phys. Rev. Lett. 83, 5290 (1999); A. San Miguel et al., Phys. Rev. B 65, 054109 (2002). 
[27] M. Wilson and P. F. McMillan, Phys. Rev. Lett. 90, 135703 (2003).

[28] D. Alfè, M. J. Gillan, M. D. Towler and R. J. Needs, Phys. Rev. B 70, 214102 (2004).

[29] F. H. Stillinger and T. A. Weber, Phys. Rev. B 31, 5262
(1985).

[30] J. Z. Hu, L. D. Merkle, C. S. Menoni and I. L. Spain, Phys. Rev. B 34, 4679 (1986). 